International Mathematical Forum, Vol. 9, 2014, no. 34, 1677 - 1693

HIKARI Ltd, www.m-hikari.com

http://dx.doi.org/10.12988/imf.2014.4482

\title{
Generating Functions of Special Triple Hypergeometric Functions
}

\author{
B. S. Desale \\ University Department of Mathematics \\ University of Mumbai \\ Mumbai 400 098, India \\ G. A. Qashash \\ Department of Mathematics \\ Faculty of Education \\ Loder-Aden University, Yemen
}

Copyright (c) 2014 B. S. Desale \& G. A. Qashash. This is an open access article distributed under the Creative Commons Attribution License, which permits unrestricted use, distribution, and reproduction in any medium, provided the original work is properly cited.

\begin{abstract}
In this paper we have obtained some generating relations involving Appell's, Exton's, Horn's, Lauricella's and Saran's functions. We obtained these relation by using integral representation of Exton's functions $X_{11}, X_{12}, \ldots, X_{20}$ in terms of Laplace's integral of several variables. Through the development of these new generating relations, we recover some Exton's, Srivastva's and Karlsson's results.
\end{abstract}

Mathematics Subject Classification: 33C65, 33C20, 33C15

Keywords: Exton Function, Appell Functions, Laplace transform \& Triple Hypergeometric series

\section{Introduction}

The unification of generating functions has great importance in connection with ideas and principles of special functions. In this directions some important steps has been made by researchers namely Singhal and Srivastava [13], 
Chaterjea [1, 2] and Chongdar [3]. In their study Desale and Qashash [4] have obtained a new general class of generating functions for the generalized modified Laguerre polynomials $L_{n}^{(\alpha)}(x)$ by group theoretic method. Also, they have introduced the bilateral generating function for the generalized modified Laguerre and Jacobi polynomials with the help of two linear partial differential operators. Further, they continued their study and they used the group theoretic method to obtain the proper and improper partial bilateral as well as trilateral generating functions. In this consent one may refer their sequel of papers $[5,6]$.

Now continuing the work in connection with class of generating functions, we extend our ideas to obtain the new generating relations that involves between Exton's functions and hyper geometric functions, in particularly Appell's functions, Lauricella's, Horn's, Saran's and Gaussian hyper geometric functions. We used integral form of Extonn's functions (in the form of Laplace integral) to obtain the new generating relations among Exton's and hypergeometric functions.

Exton in $[9,10]$ gave integral representation of some hyper geometric functions of three variables out of which we referred here $X_{11}, X_{12}, \ldots, X_{20}{ }^{1}$ and these relations are defined as follows (cf. $[9,10])$.

$$
\begin{gathered}
X_{11}(a, b ; c, d ; x, y, z)=\sum_{m, n, p=0}^{\infty} \frac{(a)_{2 m+n}(b)_{n+2 p} x^{m} y^{n} z^{p}}{(c)_{m+p}(d)_{n} m ! n ! p !} \\
X_{12}\left(a, b ; c_{1}, c_{2}, c_{3} ; x, y, z\right)=\sum_{m, n, p=0}^{\infty} \frac{(a)_{2 m+n}(b)_{n+2 p} x^{m} y^{n} z^{p}}{\left(c_{1}\right)_{m}\left(c_{2}\right)_{n}\left(c_{3}\right)_{p} m ! n ! p !} \\
X_{13}(a, b, c ; d ; x, y, z)=\sum_{m, n, p=0}^{\infty} \frac{(a)_{2 m+n}(b)_{n+p}(c)_{p} x^{m} y^{n} z^{p}}{(d)_{m+n+p} m ! n ! p !} \\
X_{14}\left(a, b, c ; d, d^{\prime} ; x, y, z\right)=\sum_{m, n, p=0}^{\infty} \frac{(a)_{2 m+n}(b)_{n+p}(c)_{p} x^{m} y^{n} z^{p}}{(d)_{m+n}\left(d^{\prime}\right)_{p} m ! n ! p !} \\
X_{15}\left(a, b, c ; d, d^{\prime} ; x, y, z\right)=\sum_{m, n, p=0}^{\infty} \frac{(a)_{2 m+n}(b)_{n+p}(c)_{p} x^{m} y^{n} z^{p}}{(d)_{m}\left(d^{\prime}\right)_{n+p} m ! n ! p !} \\
X_{16}\left(a, b, c ; d, d^{\prime} ; x, y, z\right)=\sum_{m, n, p=0}^{\infty} \frac{(a)_{2 m+n}(b)_{n+p}(c)_{p} x^{m} y^{n} z^{p}}{(d)_{m+p}\left(d^{\prime}\right)_{n} m ! n ! p !} \\
X_{17}\left(a, b, c ; d_{1}, d_{2}, d_{3} ; x, y, z\right)=\sum_{m, n, p=0}^{\infty} \frac{(a)_{2 m+n}(b)_{n+p}(c)_{p} x^{m} y^{n} z^{p}}{\left(d_{1}\right)_{m}\left(d_{2}\right)_{n}\left(d_{3}\right)_{p} m ! n ! p !}
\end{gathered}
$$

\footnotetext{
${ }^{1}$ In our earlier work, we determined the generating relations by integral transformation of $X_{1}, X_{2}, \ldots, X_{10}$. More details about our results may be referred from [7]
} 


$$
\begin{gathered}
X_{18}\left(a, b, b^{\prime}, c ; d ; x, y, z\right)=\sum_{m, n, p=0}^{\infty} \frac{(a)_{2 m+n}(b)_{n}\left(b^{\prime}\right)_{p}(c)_{p} x^{m} y^{n} z^{p}}{(d)_{m+n+p} m ! n ! p !} \\
X_{19}\left(a, b, b^{\prime}, c ; d, d^{\prime} ; x, y, z\right)=\sum_{m, n, p=0}^{\infty} \frac{(a)_{2 m+n}(b)_{n}\left(b^{\prime}\right)_{p}(c)_{p} x^{m} y^{n} z^{p}}{(d)_{m}\left(d^{\prime}\right)_{n+p} m ! n ! p !} \\
X_{20}\left(a, b, b^{\prime}, c ; d, d^{\prime} ; x, y, z\right)=\sum_{m, n, p=0}^{\infty} \frac{(a)_{2 m+n}(b)_{n}\left(b^{\prime}\right)_{p}(c)_{p} x^{m} y^{n} z^{p}}{(d)_{m+n}\left(d^{\prime}\right)_{n} m ! n ! p !}
\end{gathered}
$$

The integral representation of these Exton functions in terms of Laplace integral are given in the following section.

\section{Integral Representation of Exton Functions}

Here, we expressed Exton functions $X_{11}, X_{12}, \ldots, X_{20}$ in terms of multiple integrals with the help of Laplace transform. Following are these integral representation of Exton functions.

$$
\begin{aligned}
& X_{11}(a, b ; c, d ; x, y, z)=\frac{1}{\Gamma(a) \Gamma(b)} \int_{0}^{\infty} \int_{0}^{\infty} \exp (-s-t) s^{a-1} t^{b-1} \\
& { }_{0} F_{1}\left(-; c ; x s^{2}+z t^{2}\right)_{0} F_{1}(-; d ; y s t) d s d t \\
& X_{12}\left(a, b ; c_{1}, c_{2}, c_{3} ; x, y, z\right)=\frac{1}{\Gamma(a) \Gamma(b)} \int_{0}^{\infty} \int_{0}^{\infty} \exp (-s-t) s^{a-1} t^{b-1} \\
& { }_{0} F_{1}\left(-; c_{1} ; x s^{2}\right)_{0} F_{1}\left(-; c_{2} ; y s t\right)_{0} F_{1}\left(-; c_{3} ; z s^{2}\right) d s d t \\
& X_{13}(a, b, c ; d ; x, y, z)=\frac{1}{\Gamma(a) \Gamma(b) \Gamma(c)} \int_{0}^{\infty} \int_{0}^{\infty} \int_{0}^{\infty} \exp (-s-t-u) \\
& \cdot s^{a-1} t^{b-1} u^{c-1} \cdot{ }_{0} F_{1}\left(-; d ; x s^{2}+y s t+z t u\right) d s d t d u, \\
& X_{14}\left(a, b, c ; d, d^{\prime} ; x, y, z\right)=\frac{1}{\Gamma(a) \Gamma(b)} \int_{0}^{\infty} \int_{0}^{\infty} \exp (-s-t) s^{a-1} t^{b-1} \\
& { }_{0} F_{1}\left(-; d ; x s^{2}+y s t\right)_{1} F_{1}\left(c ; d^{\prime} ; z t\right) d s d t
\end{aligned}
$$




$$
\begin{aligned}
& X_{15}\left(a, b, c ; d, d^{\prime} ; x, y, z\right)=\frac{1}{\Gamma(a) \Gamma(c)} \int_{0}^{\infty} \int_{0}^{\infty} \exp (-s-t) \\
& \cdot s^{a-1} t^{c-1}{ }_{0} F_{1}\left(-; d ; x s^{2}\right){ }_{1} F_{1}\left(b ; d^{\prime} ; y s+z t\right) d s d t, \\
& X_{16}\left(a, b, c ; d, d^{\prime} ; x, y, z\right)=\frac{1}{\Gamma(a) \Gamma(b) \Gamma(c)} \int_{0}^{\infty} \int_{0}^{\infty} \int_{0}^{\infty} \exp (-s-t-u) \\
& \cdot s^{a-1} t^{b-1} u^{c-1}{ }_{0} F_{1}\left(-; d ; x s^{2}+z t u\right)_{0} F_{1}\left(-; d^{\prime} ; y s t\right) d s d t d u \text {, } \\
& X_{17}\left(a, b, c ; d_{1}, d_{2}, d_{3} ; x, y, z\right)=\frac{1}{\Gamma(a) \Gamma(c)} \int_{0}^{\infty} \int_{0}^{\infty} \exp (-s-t) s^{a-1} t^{c-1} \\
& { }_{0} F_{1}\left(-; d_{1} ; x s^{2}\right) \Psi_{2}\left(b ; d_{2}, d_{3} ; y s+z t\right) d s d t \text {, } \\
& X_{18}\left(a, b, b^{\prime}, c ; d ; x, y, z\right)=\frac{1}{\Gamma(a) \Gamma(b) \Gamma\left(b^{\prime}\right) \Gamma(c)} \int_{0}^{\infty} \int_{0}^{\infty} \int_{0}^{\infty} \int_{0}^{\infty} e^{(-s-t-u-v)} \\
& \cdot s^{a-1} t^{b-1} u^{b^{\prime}-1} v^{c-1}{ }_{0} F_{1}\left(-; d ; x s^{2}+y s t+z u v\right) d s d t d u d v \\
& X_{19}\left(a, b, b^{\prime}, c ; d, d^{\prime} ; x, y, z\right)=\frac{1}{\Gamma(a) \Gamma(c)} \int_{0}^{\infty} \int_{0}^{\infty} \exp (-s-t) \\
& \cdot s^{a-1} t^{c-1}{ }_{0} F_{1}\left(-; d ; x s^{2}\right) \Phi_{2}\left(b, b^{\prime} ; d^{\prime} ; y s+z t\right) d s d t, \\
& X_{20}\left(a, b, b^{\prime}, c ; d, d^{\prime} ; x, y, z\right)=\frac{1}{\Gamma(a) \Gamma\left(b^{\prime}\right) \Gamma(c)} \int_{0}^{\infty} \int_{0}^{\infty} \int_{0}^{\infty} e^{(-s-t-u)} s^{a-1} \\
& \cdot t^{b^{\prime}-1} u^{c-1}{ }_{0} F_{1}\left(-; d ; x s^{2}+z t u\right)_{1} F_{1}\left(b ; d^{\prime} ; y s\right) d s d t d u \text {. }
\end{aligned}
$$

\section{New Generating Relations}

In this section, we used the integral representation of Exton functions to determine new generating functions which are listed in the form of following 
relations (3.1) to (3.18).

$$
\begin{aligned}
& \sum_{n=0}^{\infty} \frac{w^{n}}{n !} X_{11}(\alpha+n, \beta+n ; \gamma, \delta ; x, y k, z)=(1+y)^{-\alpha}(1+k)^{-\beta} \\
& \cdot \sum_{n, r, m=0}^{\infty} \frac{(\alpha+n)_{r}(\alpha+n)_{m}(\delta)_{r+m}}{(\delta)_{r}(\delta)_{m} n ! m ! r !}\left(\frac{y}{1+y}\right)^{r}\left(\frac{k}{1+k}\right)^{m}\left(\frac{w}{(1+y)(1+k)}\right)^{n} \\
& \cdot F_{3}\left(\frac{\alpha+n+r}{2}, \frac{\beta+n+m}{2}, \frac{\alpha+n+r+1}{2},\right. \\
& \left.\frac{\beta+n+m+1}{2} ; \gamma ; \frac{4 x}{(1+y)^{2}}, \frac{4 z}{(1+k)^{2}}\right),
\end{aligned}
$$

$$
\begin{aligned}
& \sum_{n=0}^{\infty} \frac{w^{n}}{n !} X_{11}(\alpha+n, \beta+n ; \gamma, \delta ; x, y k, z)=(1+y)^{-\alpha}(1+k)^{-\beta} \\
& \cdot \sum_{n, p, q=0}^{\infty} \frac{(\alpha+n)_{2 p}(\beta+n)_{2 q}}{(\gamma)_{p+q} n ! p ! q !}\left(\frac{x}{(1+y)^{2}}\right)^{p}\left(\frac{z}{(1+k)^{2}}\right)^{q}\left(\frac{w}{(1+y)(1+k)}\right)^{n} \\
& \cdot F_{2}\left(\delta, \alpha+n+2 p, \beta+n+2 q ; \delta, \delta ; \frac{y}{1+y}, \frac{k}{1+k}\right) \\
& \quad \sum_{n=0}^{\infty} \frac{w^{n}}{n !} X_{12}\left(\alpha+n, \beta+n ; \gamma, \delta, \lambda ; x^{2}, y, z^{2}\right) \\
& =(1+2 x)^{-\alpha}(1+2 z)^{-\beta} \sum_{n=0}^{\infty} \frac{1}{n !}\left(\frac{w}{(1+2 x)(1+2 z)}\right)^{n} \\
& \cdot F_{k}\left(\gamma-\frac{1}{2}, \beta+n, \beta+n, \alpha+n, \lambda-\frac{1}{2}, \alpha+n ; 2 \gamma-1,2 \lambda-1, \delta\right. \\
& \left.\quad \frac{4 x}{(1+2 x)}, \frac{4 z}{(1+2 z)}, \frac{y}{(1+2 x)(1+2 z)}\right)
\end{aligned}
$$$$
\sum_{n=0}^{\infty} \frac{w^{n}}{n !} X_{12}\left(\alpha+n, \beta+n ; \gamma, \delta, \lambda ; x^{2}, y, z\right)=(1+2 x)^{-\alpha} \sum_{n=0}^{\infty} \frac{1}{n !}\left(\frac{w}{1+2 x}\right)^{n}
$$$$
\cdot X_{17}\left(\beta+n, \alpha+n, \gamma-\frac{1}{2} ; \lambda, \delta, 2 \gamma-1 ; z, \frac{y}{(1+2 x)}, \frac{4 x}{(1+2 x)}\right) \text {, }
$$

$$
\begin{aligned}
& \sum_{n=0}^{\infty} \frac{w^{n}}{n !} X_{12}\left(\alpha+n, \beta+n ; \gamma, \delta, \lambda ; x, y, z^{2}\right)=(1+2 z)^{-\beta} \sum_{n=0}^{\infty} \frac{1}{n !}\left(\frac{w}{1+2 z}\right)^{n} \\
& \cdot X_{17}\left(\alpha+n, \beta+n, \lambda-\frac{1}{2} ; \gamma, \delta, 2 \lambda-1 ; x, \frac{y}{(1+2 z)}, \frac{4 z}{(1+2 z)}\right)
\end{aligned}
$$




$$
\begin{aligned}
& \sum_{n=0}^{\infty} \frac{w^{n}}{n !} X_{14}(\alpha+n, \beta+n, \gamma ; \delta, \epsilon ; x, y, z)=(1-z)^{-\beta} \sum_{n=0}^{\infty} \frac{1}{n !}\left(\frac{w}{1-z}\right)^{n} \\
& \cdot X_{14}\left(\alpha+n, \beta+n, \epsilon-\gamma ; \delta, \epsilon ; x, \frac{y}{(1-z)}, \frac{z}{(z-1)}\right) \\
& \sum_{n=0}^{\infty} \frac{w^{n}}{n !} X_{14}(\alpha+n, \beta+n, \gamma ; \delta, \gamma ; x, y, z)=(1-z)^{-\beta} \sum_{n=0}^{\infty} \frac{1}{n !}\left(\frac{w}{1-z}\right)^{n} \\
& \cdot H_{3}\left(\alpha+n, \beta+n ; \delta ; x, \frac{y}{(1-z)}\right),
\end{aligned}
$$

$$
\begin{aligned}
& \sum_{n=0}^{\infty} \frac{w^{n}}{n !} X_{15}\left(\alpha+n, \beta, \gamma+n ; \delta, \epsilon ; x^{2}, y, z\right)=(1+2 x)^{-\alpha} \sum_{n=0}^{\infty} \frac{1}{n !}\left(\frac{w}{1+2 x}\right)^{n} \\
& \cdot F_{M}\left(\delta-\frac{1}{2}, \beta, \beta, \alpha+n, \gamma+n, \alpha+n ; 2 \delta-1, \epsilon, \epsilon ; \frac{4 x}{(1+2 x)}, z, \frac{y}{(1+2 x)}\right)
\end{aligned}
$$

$$
\begin{aligned}
& \sum_{n=0}^{\infty} \frac{w^{n}}{n !} X_{15}(\alpha+n, \beta, \gamma+n ; \delta, \beta ; x, y, z)=(1-y)^{-\alpha}(1-z)^{-\gamma} \sum_{n=0}^{\infty} \frac{1}{n !} \\
& \cdot\left(\frac{w}{(1-y)(1-z)}\right)^{n}{ }_{2} F_{1}\left(\frac{\alpha+n}{2}, \frac{\alpha+n+1}{2} ; \delta ; \frac{4 x}{(1-y)^{2}}\right)
\end{aligned}
$$

$$
\begin{aligned}
& \sum_{n=0}^{\infty} \frac{w^{n}}{n !} X_{15}\left(\alpha+n, \beta, \gamma+n ; \delta, \beta ; x^{2}, y, z\right) \\
& =(1+2 x-y)^{-\alpha}(1-z)^{-\gamma} \sum_{n=0}^{\infty} \frac{1}{n !}\left(\frac{w}{(1+2 x-y)(1-z)}\right)^{n} \\
& { }_{2} F_{1}\left(\alpha+n, \delta-\frac{1}{2} ; 2 \delta-1 ; \frac{4 x}{(1+2 x-y)}\right)
\end{aligned}
$$$$
\sum_{n=0}^{\infty} \frac{w^{n}}{n !} X_{17}\left(\alpha+n, \beta, \gamma+n ; \delta_{1}, \delta_{2}, \delta_{3} ; x^{2}, y, z\right)
$$$$
=(1+2 x)^{-\alpha} \sum_{n=0}^{\infty} \frac{1}{n !}\left(\frac{w}{(1+2 x)}\right)^{n}
$$$$
\cdot F_{K}\left(\delta_{1}-\frac{1}{2}, \beta, \beta, \alpha+n, \gamma+n, \alpha+n ; 2 \delta_{1}-1, \delta_{3}, \delta_{2} ; \frac{4 x}{1+2 x}, z, \frac{y}{1+2 x}\right) \text {, }
$$ 


$$
\begin{aligned}
& \sum_{n=0}^{\infty} \frac{w^{n}}{n !} X_{17}(\alpha+n, \beta, \gamma+n ; \delta, \beta, \beta ; x, y, z)=(1-y)^{-\alpha}(1-z)^{-\gamma} \sum_{n=0}^{\infty} \frac{1}{n !} \\
& \cdot\left(\frac{w}{(1-y)(1-z)}\right)^{n} H_{4}\left(\alpha+n, \gamma+n ; \delta, \beta ; \frac{x}{(1-y)^{2}}, \frac{y z}{(1-y)(1-z)}\right)
\end{aligned}
$$

$$
\begin{aligned}
& \sum_{n=0}^{\infty} \frac{w^{n}}{n !} X_{17}\left(\alpha+n, \beta, \gamma+n ; \delta, \beta, \beta ; x^{2}, y, z\right) \\
& =(1+2 x-y)^{-\alpha}(1-z)^{-\gamma} \sum_{n=0}^{\infty} \frac{1}{n !}\left(\frac{w}{(1+2 x-y)(1-z)}\right)^{n} \\
& F_{2}\left(\alpha+n, \delta-\frac{1}{2}, \gamma+n ; 2 \delta-1, \beta ; \frac{4 x}{(1+2 x-y)}, \frac{y z}{(1+2 x-y)(1-z)}\right),
\end{aligned}
$$

$$
\begin{aligned}
& \sum_{n=0}^{\infty} \frac{w^{n}}{n !} X_{19}\left(\alpha+n, \beta_{1}, \beta_{2}, \gamma+n ; \delta_{1}, \delta_{2} ; x^{2}, y, z\right) \\
& =(1+2 x)^{-\alpha} \sum_{n=0}^{\infty} \frac{1}{n !}\left(\frac{w}{(1+2 x)}\right)^{n} F_{N}\left(\delta_{1}-\frac{1}{2}, \beta_{2}, \beta_{1}, \alpha+n, \alpha+n, \alpha+n ;\right. \\
& \left.2 \delta_{1}-1, \delta_{2}, \delta_{2} ; \frac{4 x}{(1+2 x)}, z, \frac{y}{(1+2 x)}\right),
\end{aligned}
$$

$$
\begin{aligned}
& \sum_{n=0}^{\infty} \frac{w^{n}}{n !} X_{19}\left(\alpha+n, \beta, \delta_{2}-\beta, \gamma+n ; \delta_{1}, \delta_{2} ; x, y, z\right)=(1-z)^{-\alpha} \\
& \cdot \sum_{n=0}^{\infty} \frac{1}{n !}\left(\frac{w}{(1-z)}\right)^{n} X_{15}\left(\alpha+n, \beta, \gamma+n ; \delta_{1}, \delta_{2} ; x, y, \frac{z}{(z-1)}\right)
\end{aligned}
$$

$$
\begin{aligned}
& \sum_{n=0}^{\infty} \frac{w^{n}}{n !} X_{19}\left(\alpha+n, \beta, \delta_{2}-\beta, \gamma+n ; \delta_{1}, \delta_{2} ; x^{2}, y, z\right) \\
& =(1+2 x)^{-\alpha}(1-z)^{-\gamma} \sum_{n=0}^{\infty} \frac{1}{n !}\left(\frac{w}{(1+2 x)(1-z)}\right)^{n} \\
& \cdot F_{M}\left(\delta_{1}-\frac{1}{2}, \beta, \beta, \alpha+n, \alpha+n, \alpha+n ; 2 \delta_{1}-1, \delta_{2}, \delta_{2} ; \frac{4 x}{(1+2 x)}, \frac{z}{(z-1)}, \frac{y}{(1+2 x)}\right),
\end{aligned}
$$




$$
\begin{aligned}
& \sum_{n=0}^{\infty} \frac{w^{n}}{n !} X_{20}\left(\alpha+n, \beta_{1}, \beta_{2}+n, \gamma+n ; \delta_{1}, \delta_{2} ; x, y, z\right) \\
& =(1-y)^{-\alpha} \sum_{n=0}^{\infty} \frac{1}{n !}\left(\frac{w}{(1-y)}\right)^{n} \\
& \cdot X_{20}\left(\alpha+n, \delta_{2}-\beta_{1}, \beta_{2}+n, \gamma+n ; \delta_{1}, \delta_{2} ; \frac{x}{(1-y)^{2}}, \frac{y}{(y-1)}, z\right) \\
& \quad \sum_{n=0}^{\infty} \frac{w^{n}}{n !} X_{20}(\alpha+n, \epsilon, \beta+n, \gamma+n ; \delta, \epsilon ; x, y, z) \\
& =(1-y)^{-\alpha} \sum_{n=0}^{\infty} \frac{1}{n !}\left(\frac{w}{(1-y)}\right)^{n} \\
& \quad F_{3}\left(\frac{\alpha+n}{2}, \beta+n, \frac{\alpha+n+1}{2}, \gamma+n ; \delta ; \frac{4 x}{(1-y)^{2}}, z\right)
\end{aligned}
$$

In above relations $F_{1}, F_{2}, F_{3}$ are the Appell functions (cf. [14], [15]), ${ }_{2} F_{1}$ is called Gaussian hypergeometric function (cf. [15], pp. 13(1) and pp. 18(17)), $H_{3}, H_{4}$ are Horn functions (see.[15], pp. 24(6-15) and [14], pp. 33(22-31)) and $F_{K}, F_{M}, F_{N}$ are Saran functions of three variables (cf. [14], pp. 67, [15])

\section{Proof of Generating relations}

To prove the above relations, we need the following formulae (cf. [14], [15], [8] and $[12])$.

$$
\begin{gathered}
\sum_{m, n=0}^{\infty} f(m+n) \frac{x^{m}}{m !} \frac{y^{n}}{n !}=\sum_{N=0}^{\infty} f(N) \frac{(x+y)^{N}}{N !}, \\
\Psi_{2}(c ; c, c ; x, y)=\exp (x+y){ }_{0} F_{1}(-; c ; x y), \\
\Phi_{2}(a, c-a ; c ; x, y)=\exp (y){ }_{1} F_{1}(a ; c ; x-y), \\
{ }_{1} F_{1}(a ; c ; x)=\exp (x){ }_{1} F_{1}(c-a ; c ;-x), \\
{ }_{0} F_{1}\left(-; c ; x^{2}\right)=\exp (-2 x){ }_{1} F_{1}\left(c-\frac{1}{2} ; 2 c-1 ; 4 x\right), \\
(\lambda)_{n}=\frac{\Gamma(\lambda+n)}{\Gamma(\lambda)}, \quad \lambda \neq 0,-1,-2, \ldots,
\end{gathered}
$$




$$
\begin{gathered}
(\lambda)_{m+n}=(\lambda)_{m}(\lambda+m)_{n}, \\
(\lambda)_{2 n}=2^{2 n}\left(\frac{\lambda}{2}\right)_{n}\left(\frac{\lambda+1}{2}\right)_{n}, \quad n=0,1,2, \ldots, \\
(n-k) !=\frac{(-1)^{k} n !}{(-n)_{k}}, \quad o \leq k \leq n, \\
L\left\{t^{2 \sigma-1}{ }_{m} F_{n}\left(\alpha_{1}, \ldots, \alpha_{m} ; \beta_{1}, \ldots, \beta_{n} ; \lambda^{2} t^{2}\right)\right\}=\Gamma(2 \sigma) p^{-2 \sigma} \\
m+2 F_{n}\left(\alpha_{1}, \ldots, \alpha_{n}, \frac{\sigma}{2}, \frac{\sigma+1}{2} ; \beta_{1}, \ldots, \beta_{n} ; 4 \lambda^{2} p^{-2}\right) \\
L\left\{t^{\gamma-1}{ }_{1} F_{1}(\alpha, \gamma ; \lambda t)\right\}=\Gamma(\gamma) p^{\alpha-\gamma}(p-\lambda)^{-\alpha}, \\
\operatorname{Re}(\gamma)>0, \operatorname{Re}(p)>0, \operatorname{Re}(\lambda)>0 . \\
L\left\{t^{v}\right\}=\Gamma(v+1) p^{-v-1}, \quad \operatorname{Re}(v)>-1, \operatorname{Re}(p)>0 . \\
L\left\{x_{1}^{\mu} F_{1}\left(a_{1} ; b_{1} ; \sigma x\right)_{1} F_{1}(a ; b ; \omega x)\right\} \\
=\Gamma(\mu+1) p^{-\mu-1} F_{2}\left(\mu+1, a, a_{1} ; b, b_{1} ; \frac{\omega}{p}, \frac{\sigma}{p}\right) \\
\operatorname{Re}(\mu)>-1, \operatorname{Re}(p-\sigma), \operatorname{Re}(p-\omega), \operatorname{Re}(p-\sigma-\omega)>0 .
\end{gathered}
$$

Where $L$ is the Laplace transform, $\Phi_{2}, \Psi_{2}$ are Humbert's functions, ${ }_{1} F_{1}$ is Kummer's function (or confluent hypergeometric function) and ${ }_{m} F_{n}$ is Generalized hypergeometric function.

\section{Proof of relation 3.1:}

Let us denote the left hand side of (3.1) by $A$, using (2.1)

$$
\begin{aligned}
A= & \sum_{n=0}^{\infty} \frac{w^{n}}{n ! \Gamma(\alpha+n) \Gamma(\beta+n)} \int_{0}^{\infty} \int_{0}^{\infty} \exp (-s-t) s^{\alpha+n-1} t^{\beta+n-1} \\
& \cdot{ }_{0} F_{1}\left(-; \gamma ; x s^{2}+z t^{2}\right)_{0} F_{1}(-; \delta ; y k s t) d s d t
\end{aligned}
$$

by using (4.1) and (4.2), we get

$$
\begin{aligned}
A= & \sum_{n, p, q=0}^{\infty} \frac{w^{n} x^{p} z^{q}}{(\gamma)_{p+q} n ! p ! q ! \Gamma(\alpha+n) \Gamma(\beta+n)} \int_{0}^{\infty} \int_{0}^{\infty} e^{(-s(1+y))} e^{(-t(1+k))} \\
& \cdot s^{\alpha+n+2 p-1} t^{\beta+n+2 q-1} \Psi_{2}(\delta ; \delta, \delta ; y s, k t) d s d t
\end{aligned}
$$


The function $\Psi_{2}$ which appears in above eqaution can be replaced by its series form and then interchanging the order of the summation and integral sign which is permissible here, we get

$$
\begin{aligned}
& A=\sum_{n, p, q, r, m=0}^{\infty} \frac{(\delta)_{r+m} w^{n} x^{p} z^{q} y^{r} k^{m}}{(\gamma)_{p+q}(\delta)_{r}(\delta)_{m} n ! p ! q ! r ! m ! \Gamma(\alpha+n) \Gamma(\beta+n)} \int_{0}^{\infty} \int_{0}^{\infty} e^{(-s(1+y))} \\
& \cdot e^{(-t(1+k))} s^{\alpha+n+2 p+r-1} t^{\beta+n+2 q+m-1} d s d t
\end{aligned}
$$

Now, we use sequentially (4.12), (4.6), (4.7) and (4.8); and then simplified by using series manipulation, which complete the proof of relation (3.1).

Remark 1. The proof of all remaining relations runs in the same way, considering the appropriate integral representation and Laplace transform during the proof.

\section{Special Cases}

Some generating relations, which are belived to be new, can be established in this section as the special cases of the results were obtained in previous section.

1. If we put $z=0$ in (3.1), then we have generating relation between Horn's function $H_{4}$ and Exton's function $X_{17}$.

$$
\begin{aligned}
& \sum_{n=0}^{\infty} \frac{w^{n}}{n !} H_{4}(\alpha+n, \beta+n ; \gamma, \delta ; x, y k)=(1+y)^{-\alpha}(1+k)^{-\beta} \sum_{n=0}^{\infty} \frac{1}{n !} \\
& \cdot\left(\frac{w}{(1+y)(1+k)}\right)^{n} X_{17}\left(\alpha+n, \delta, \beta+n ; \gamma, \delta, \delta ; \frac{x}{(1+y)^{2}}, \frac{y}{1+y}, \frac{k}{1+k}\right)
\end{aligned}
$$

2. Now assign the value to $x$ and $z$ to be zero in (3.2), we get the relation between Gaussian hypergeometric function and Appell's function.

$$
\begin{aligned}
& \sum_{n=0}^{\infty} \frac{w^{n}}{n !}{ }_{2} F_{1}(\alpha+n, \beta+n ; \delta ; y k)=(1+y)^{-\alpha}(1+k)^{-\beta} \\
& \cdot \sum_{n=0}^{\infty} \frac{1}{n !}\left(\frac{w}{(1+y)(1+k)}\right)^{n} F_{2}\left(\delta, \alpha+n, \beta+n ; \delta, \delta ; \frac{y}{1+y}, \frac{k}{1+k}\right) .
\end{aligned}
$$

3. Put $x=0$ in equations (3.3), (3.5), (3.6), (3.7), (3.12), and (3.15), we 
obtained the following generating relations.

$$
\begin{aligned}
& \sum_{n=0}^{\infty} \frac{w^{n}}{n !} H_{4}\left(\beta+n, \alpha+n ; \lambda, \delta ; z^{2}, y\right)=(1+2 z)^{-\beta} \sum_{n=0}^{\infty} \frac{1}{n !}\left(\frac{w}{(1+2 z)}\right)^{n} \\
& \cdot F_{2}\left(\alpha+n, \lambda-\frac{1}{2}, \alpha+n ; 2 \lambda-1, \delta ; \frac{4 z}{(1+2 z)}, \frac{y}{(1+2 z)}\right) \\
& \sum_{n=0}^{\infty} \frac{w^{n}}{n !} H_{4}\left(\beta+n, \alpha+n ; \lambda, \delta ; z^{2}, y\right)=(1+2 z)^{-\beta} \sum_{n=0}^{\infty} \frac{1}{n !}\left(\frac{w}{1+2 z}\right)^{n} \\
& \cdot F_{2}\left(\beta+n, \alpha+n, \lambda-\frac{1}{2} ; \delta, 2 \lambda-1 ; \frac{y}{(1+2 z)}, \frac{4 z}{(1+2 z)}\right) \\
& \sum_{n=0}^{\infty} \frac{w^{n}}{n !} F_{2}(\beta+n, \alpha+n, \gamma ; \delta, \epsilon ; y, z)=(1-z)^{-\beta} \sum_{n=0}^{\infty} \frac{1}{n !} \\
& \cdot\left(\frac{w}{1-z}\right)^{n} F_{2}\left(\beta+n, \alpha+n, \epsilon-\gamma ; \delta, \epsilon ; \frac{y}{(1-z)}, \frac{z}{(z-1)}\right) \\
& \sum_{n=0}^{\infty} \frac{w^{n}}{n !} F_{2}(\beta+n, \alpha+n, \gamma ; \delta, \gamma ; y, z)=(1-z)^{-\beta} \\
& \cdot \sum_{n=0}^{\infty} \frac{1}{n !}\left(\frac{w}{1-z}\right)^{n}{ }_{2} F_{1}\left(\alpha+n, \beta+n ; \delta ; \frac{y}{(1-z)}\right) \\
& \sum_{n=0}^{\infty} \frac{w^{n}}{n !} F_{2}(\beta, \alpha+n, \gamma+n ; \beta, \beta ; y, z)=(1-y)^{-\alpha}(1-z)^{-\gamma} \sum_{n=0}^{\infty} \frac{1}{n !} \\
& \cdot\left(\frac{w}{(1-y)(1-z)}\right)^{n}{ }_{2} F_{1}\left(\alpha+n, \gamma+n ; \beta ; \frac{y z}{(1-y)(1-z)}\right) \\
& \sum_{n=0}^{\infty} \frac{w^{n}}{n !} F_{3}\left(\alpha+n, \delta_{2}-\beta, \beta, \gamma+n ; \delta_{2} ; y, z\right)=(1-z)^{-\gamma} \sum_{n=0}^{\infty} \frac{1}{n !} \\
& \cdot\left(\frac{w}{(1-z)}\right)^{n} F_{1}\left(\beta, \alpha+n, \gamma+n ; \delta_{2} ; y, \frac{z}{(z-1)}\right)
\end{aligned}
$$


4. Now substituting $z=0$ in (3.4), (3.8), (3.9), (3.10), (3.11), (3.14), (3.17), and (3.18), we obtain the following relations.

$$
\begin{aligned}
& \sum_{n=0}^{\infty} \frac{w^{n}}{n !} H_{4}\left(\alpha+n, \beta+n ; \gamma, \delta ; x^{2}, y\right)=(1+2 x)^{-\alpha} \sum_{n=0}^{\infty} \frac{1}{n !}\left(\frac{w}{1+2 x}\right)^{n} \\
& F_{2}\left(\alpha+n, \beta+n, \gamma-\frac{1}{2} ; \delta, 2 \gamma-1 ; \frac{y}{(1+2 x)}, \frac{4 x}{(1+2 x)}\right) \\
& \sum_{n=0}^{\infty} \frac{w^{n}}{n !} H_{4}\left(\alpha+n, \beta ; \delta, \epsilon ; x^{2}, y\right)=(1+2 x)^{-\alpha} \sum_{n=0}^{\infty} \frac{1}{n !}\left(\frac{w}{1+2 x}\right)^{n} \\
& \cdot F_{2}\left(\alpha+n, \beta, \delta-\frac{1}{2} ; \epsilon, 2 \delta-1 ; \frac{y}{(1+2 x)}, \frac{4 x}{(1+2 x)}\right) \\
& \sum_{n=0}^{\infty} \frac{w^{n}}{n !} H_{4}(\alpha+n, \beta ; \delta, \beta ; x, y)=(1-y)^{-\alpha} \sum_{n=0}^{\infty} \frac{1}{n !} \\
& \cdot\left(\frac{w}{(1-y)}\right)_{2}^{n} F_{1}\left(\frac{\alpha+n}{2}, \frac{\alpha+n+1}{2} ; \delta ; \frac{4 x}{(1-y)^{2}}\right) \\
& \sum_{n=0}^{\infty} \frac{w^{n}}{n !} H_{4}\left(\alpha+n, \beta ; \delta, \beta ; x^{2}, y\right)=(1+2 x-y)^{-\alpha} \sum_{n=0}^{\infty} \frac{1}{n !} \\
& \cdot\left(\frac{w}{(1+2 x-y)}\right)^{n}{ }_{2} F_{1}\left(\alpha+n, \delta-\frac{1}{2} ; 2 \delta-1 ; \frac{4 x}{(1+2 x-y)}\right) \\
& \sum_{n=0}^{\infty} \frac{w^{n}}{n !} H_{4}\left(\alpha+n, \beta ; \delta_{1}, \delta_{2} ; x^{2}, y\right)=(1+2 x)^{-\alpha} \sum_{n=0}^{\infty} \frac{1}{n !} \\
& \cdot\left(\frac{w}{(1+2 x)}\right)^{n} F_{2}\left(\alpha+n, \delta_{1}-\frac{1}{2}, \beta ; 2 \delta_{1}-1, \delta_{2} ; \frac{4 x}{1+2 x}, \frac{y}{1+2 x}\right) \\
& \sum_{n=0}^{\infty} \frac{w^{n}}{n !} H_{4}\left(\alpha+n, \beta_{1} ; \delta_{1}, \delta_{2} ; x^{2}, y\right)=(1+2 x)^{-\alpha} \sum_{n=0}^{\infty} \frac{1}{n !} \\
& \cdot\left(\frac{w}{(1+2 x)}\right)^{n} F_{2}\left(\alpha+n, \delta_{1}-\frac{1}{2}, \beta_{1} ; 2 \delta_{1}-1, \delta_{2} ; \frac{4 x}{(1+2 x)}, \frac{y}{(1+2 x)}\right)
\end{aligned}
$$




$$
\begin{aligned}
& \sum_{n=0}^{\infty} \frac{w^{n}}{n !} H_{4}\left(\alpha+n, \beta_{1} ; \delta_{1}, \delta_{2} ; x, y\right)=(1-y)^{-\alpha} \sum_{n=0}^{\infty} \frac{1}{n !} \\
& \cdot\left(\frac{w}{(1-y)}\right)^{n} H_{4}\left(\alpha+n, \delta_{2}-\beta_{1} ; \delta_{1}, \delta_{2} ; \frac{x}{(1-y)^{2}}, \frac{y}{(y-1)}\right)
\end{aligned}
$$

$$
\begin{aligned}
& \sum_{n=0}^{\infty} \frac{w^{n}}{n !} H_{4}(\alpha+n, \epsilon ; \delta, \epsilon ; x, y)=(1-y)^{-\alpha} \sum_{n=0}^{\infty} \frac{1}{n !} \cdot\left(\frac{w}{(1-y)}\right)^{n} \\
& { }_{2} F_{1}\left(\frac{\alpha+n}{2}, \frac{\alpha+n+1}{2} ; \delta ; \frac{4 x}{(1-y)^{2}}\right)
\end{aligned}
$$

5. Here, we determine some new relations, which are obtained by substituting $n=0$ into the equations (3.1), (3.2), (3.10), (3.13), and (3.16).

$$
\begin{aligned}
& X_{11}(\alpha, \beta ; \gamma, \delta ; x, y k, z)=(1+y)^{-\alpha}(1+k)^{-\beta} \sum_{r, m=0}^{\infty} \frac{(\alpha)_{r}(\alpha)_{m}(\delta)_{r+m}}{(\delta)_{r}(\delta)_{m} m ! r !} \\
& \cdot\left(\frac{y}{1+y}\right)^{r}\left(\frac{k}{1+k}\right)^{m} F_{3}\left(\frac{\alpha+r}{2}, \frac{\beta+m}{2}, \frac{\alpha+r+1}{2}, \frac{\beta+m+1}{2} ; \gamma ; \frac{4 x}{(1+y)^{2}}, \frac{4 z}{(1+k)^{2}}\right) \\
& X_{11}(\alpha, \beta ; \gamma, \delta ; x, y k, z)=(1+y)^{-\alpha}(1+k)^{-\beta} \sum_{p, q=0}^{\infty} \frac{(\alpha)_{2 p}(\beta)_{2 q}}{(\gamma)_{p+q} p ! q !} \\
& \cdot\left(\frac{x}{(1+y)^{2}}\right)^{p}\left(\frac{z}{(1+k)^{2}}\right)^{q} F_{2}\left(\delta, \alpha+2 p, \beta+2 q ; \delta, \delta ; \frac{y}{1+y}, \frac{k}{1+k}\right)^{-\alpha}(1-z)^{-\gamma} \\
& \quad X_{15}\left(\alpha, \beta, \gamma ; \delta, \beta ; x^{2}, y, z\right)=(1+2 x-y)^{-\alpha}(1) \\
& \quad F_{2}\left(\alpha, \delta-\frac{1}{2} ; 2 \delta-1 ; \frac{4 x}{(1+2 x-y)}\right) \\
& \quad X_{17}\left(\alpha, \beta, \gamma ; \delta, \beta, \beta ; x^{2}, y, z\right)=(1+2 x-y)^{-\alpha}(1-z)^{-\gamma} \\
& \quad \cdot F_{2}\left(\alpha, \delta-\frac{1}{2}, \gamma ; 2 \delta-1, \beta ; \frac{4 x}{(1+2 x-y)}, \frac{y z}{(1+2 x-y)(1-z)}\right) \\
& X_{19}\left(\alpha, \beta, \delta_{2}-\beta, \gamma ; \delta_{1}, \delta_{2} ; x^{2}, y, z\right)=(1+2 x)^{-\alpha}(1-z)^{-\gamma} \\
& \cdot F_{M}\left(\delta_{1}-\frac{1}{2}, \beta, \beta, \alpha, \alpha, \alpha ; 2 \delta_{1}-1, \delta_{2}, \delta_{2} ; \frac{4 x}{(1+2 x)}, \frac{z}{(z-1)}, \frac{y}{(1+2 x)}\right)
\end{aligned}
$$

6. The following results are the Exton's results, which are obtained by Exton in his paper (cf. [10]). We recover these result by assigning the value zero 
to $n$ into the equations $(3.3),(3.4),(3.5),(3.6),(3.7),(3.8),(3.9),(3.11)$, (3.12), (3.14), (3.15), (3.17) and (3.18), we get

$$
\begin{gathered}
X_{12}\left(\alpha, \beta ; \gamma, \delta, \lambda ; x^{2}, y, z^{2}\right)=(1+2 x)^{-\alpha}(1+2 z)^{-\beta} \\
\cdot F_{k}\left(\gamma-\frac{1}{2}, \beta, \beta, \alpha, \lambda-\frac{1}{2}, \alpha ; 2 \gamma-1,2 \lambda-1, \delta\right. \\
\left.\frac{4 x}{(1+2 x)}, \frac{4 z}{(1+2 z)}, \frac{y}{(1+2 x)(1+2 z)}\right) \\
X_{12}\left(\alpha, \beta ; \gamma, \delta, \lambda ; x^{2}, y, z\right)=(1+2 x)^{-\alpha} \\
\cdot X_{17}\left(\beta, \alpha, \gamma-\frac{1}{2} ; \lambda, \delta, 2 \gamma-1 ; z, \frac{y}{(1+2 x)}, \frac{4 x}{(1+2 x)}\right) \\
\quad X_{12}\left(\alpha, \beta ; \gamma, \delta, \lambda ; x, y, z^{2}\right)=(1+2 z)^{-\beta} \\
\cdot X_{17}\left(\alpha, \beta, \lambda-\frac{1}{2} ; \gamma, \delta, 2 \lambda-1 ; x, \frac{y}{(1+2 z)}, \frac{4 z}{(1+2 z)}\right) \\
X_{14}(\alpha, \beta, \gamma ; \delta, \epsilon ; x, y, z) \\
=(1-z)^{-\beta} X_{14}\left(\alpha, \beta, \epsilon-\gamma ; \delta, \epsilon ; x, \frac{y}{(1-z)}, \frac{z}{(z-1)}\right) \\
X_{14}(\alpha, \beta, \gamma ; \delta, \gamma ; x, y, z)=(1-z)^{-\beta} H_{3}\left(\alpha, \beta ; \delta ; x, \frac{y}{(1-z)}\right)
\end{gathered}
$$

$$
\begin{aligned}
& X_{15}\left(\alpha, \beta, \gamma ; \delta, \epsilon ; x^{2}, y, z\right)=(1+2 x)^{-\alpha} \\
& \cdot F_{M}\left(\delta-\frac{1}{2}, \beta, \beta, \alpha, \gamma, \alpha ; 2 \delta-1, \epsilon, \epsilon ; \frac{4 x}{(1+2 x)}, z, \frac{y}{(1+2 x)}\right) \\
& \quad X_{15}(\alpha, \beta, \gamma ; \delta, \beta ; x, y, z) \\
& \quad=(1-y)^{-\alpha}(1-z)^{-\gamma}{ }_{2} F_{1}\left(\frac{\alpha}{2}, \frac{\alpha+1}{2} ; \delta ; \frac{4 x}{(1-y)^{2}}\right) \\
& X_{17}\left(\alpha, \beta, \gamma ; \delta_{1}, \delta_{2}, \delta_{3} ; x^{2}, y, z\right)=(1+2 x)^{-\alpha} \\
& \cdot F_{K}\left(\delta_{1}-\frac{1}{2}, \beta, \beta, \alpha, \gamma, \alpha ; 2 \delta_{1}-1, \delta_{3}, \delta_{2} ; \frac{4 x}{1+2 x}, z, \frac{y}{1+2 x}\right)
\end{aligned}
$$

$$
\begin{aligned}
& X_{17}(\alpha, \beta, \gamma ; \delta, \beta, \beta ; x, y, z) \\
& =(1-y)^{-\alpha}(1-z)^{-\gamma} H_{4}\left(\alpha, \gamma ; \delta, \beta ; \frac{x}{(1-y)^{2}}, \frac{y z}{(1-y)(1-z)}\right)
\end{aligned}
$$




$$
\begin{aligned}
& X_{19}\left(\alpha, \beta_{1}, \beta_{2}, \gamma ; \delta_{1}, \delta_{2} ; x^{2}, y, z\right)=(1+2 x)^{-\alpha} \\
& \cdot F_{N}\left(\delta_{1}-\frac{1}{2}, \beta_{2}, \beta_{1}, \alpha, \alpha, \alpha ; 2 \delta_{1}-1, \delta_{2}, \delta_{2} ; \frac{4 x}{(1+2 x)}, z, \frac{y}{(1+2 x)}\right) \\
& \quad X_{19}\left(\alpha, \beta, \delta_{2}-\beta, \gamma ; \delta_{1}, \delta_{2} ; x, y, z\right)= \\
& \quad(1-z)^{-\alpha} X_{15}\left(\alpha, \beta, \gamma ; \delta_{1}, \delta_{2} ; x, y, \frac{z}{(z-1)}\right) \\
& \left.X_{20}\left(\alpha, \beta_{1}, \beta_{2}, \gamma ; \delta_{1}, \delta_{2} ; x, y, z\right)=(1-y)^{-\alpha}\right) \\
& \cdot X_{20}\left(\alpha, \delta_{2}-\beta_{1}, \beta_{2}, \gamma ; \delta_{1}, \delta_{2} ; \frac{x}{(1-y)^{2}}, \frac{y}{(y-1)}, z\right) \\
& X_{20}(\alpha, \epsilon, \beta, \gamma ; \delta, \epsilon ; x, y, z) \\
& =(1-y)^{-\alpha} F_{3}\left(\frac{\alpha}{2}, \beta, \frac{\alpha+1}{2}, \gamma ; \delta ; \frac{4 x}{(1-y)^{2}}, z\right)
\end{aligned}
$$

7. Following relations are the results of Srivastava's and Karlsson's. Redears may concent their book [15] for more details of these results. We recover these result by assignning the value zero to $n$ into the equations (5.2), (5.7) and (5.8).

$$
{ }_{2} F_{1}(\alpha, \beta ; \delta ; y k)=(1+y)^{-\alpha}(1+k)^{-\beta} F_{2}\left(\delta, \alpha, \beta ; \delta, \delta ; \frac{y}{1+y}, \frac{k}{1+k}\right)
$$

When we put $y=\frac{k}{1+k}$ and $x=\frac{y}{1+y}$.

$$
\begin{gathered}
F_{2}(\beta, \alpha, \gamma ; \beta, \beta ; y, z) \\
=(1-y)^{-\alpha}(1-z)^{-\gamma}{ }_{2} F_{1}\left(\alpha, \gamma ; \beta ; \frac{y z}{(1-y)(1-z)}\right) \\
F_{3}\left(\alpha, \delta_{2}-\beta, \beta, \gamma ; \delta_{2} ; y, z\right)=(1-z)^{-\gamma} F_{1}\left(\beta, \alpha, \gamma ; \delta_{2} ; y, \frac{z}{(z-1)}\right)
\end{gathered}
$$

\section{Conclusion}

We used the Laplace integral representation of Exton's functions given by (1.1) to (1.10) to determine the new generating relations (3.1) to (3.18). Many of these results are the relations between Appell's functions $F_{1}, F_{2}, F_{3}$; Horn's functions $H_{3}, H_{4}$; Saran's functions $F_{K}, F_{M}, F_{N}$ and Gaussian hypergeometric function ${ }_{2} F_{1}$. One of the special case of $(5.1)$ is the generating relation between Horn's and Exton's functions. The generating relation (5.2) is the relation between Gaussian hypergeometric function and Appell's functions. Also, we have seen in the section of special cases many of the results from section 3 reduces to Exton's functions for $n=0$. 


\section{References}

[1] S. K. Chatterjea, Unification of a class of bilateral generating relations for certain special functions, Bull. Cal. Math. Soc., 67 (1975), pp. 115-127.

[2] S. K. Chatterjea, An extension of a class of bilateral generating functions for certain special functions, Bull. Inst. Math. Acad. Sinica 5(2) (1977).

[3] A. K. Chongdar, On the unification of a class of bilateral generating functions for certain special functions, Tamkang Jour. Math., 18 (3) (1987), pp. 53-59.

[4] B. S. Desale and G. A. Qashash, A General Class of Generating Functions of Lagurre Polynomials, Journal of Inequality and Special Functions, 2(2) (2011), pp. 1-7.

[5] B. S. Desale and G. A. Qashash, Improper Partial Bilateral Generating Functions for Some Orthogonal Polynomials, Int. Journal of Math. Analysis, 5, no 47 (2011), pp. 2321-2328.

[6] B. S. Desale and G. A. Qashash, Trilateral Generating Function for Hermite, Jacobi and Bessel Polynomials, Int. Journal of Math. Analysis, 5, no 47 (2011), pp. 2329-2335.

[7] B. S. Desale, G. A. Qashash, Generating Relations Between Extons Functions and Triple Hypergeometric Functions, Far East Jr of Applied Mathematical, Vol 78 No. 2, (2011), pp. 91-114.

[8] A. Erdelyi, W. Magnus, F. Oberhettinger and F. G. Tricomi, Tables of integral Transforms, vol 1 and 2, McGraw-Hill, New York, (1954).

[9] H. Exton, Multiple Hypergeometric Functions and Applications, Ellis Horwood Ltd., Chichester, U. K.(1976).

[10] H. Exton, Hypergeometric functions of three variables, Journal of Indian Acad. Math. 4, No. 2 (1982), pp. 113-119.

[11] M. G. Kendall and A. Stuart, The Advanced Theory of Statistics, Griffin, London, (1958).

[12] E. D. Rainville, Special Functions, Chelsea Publication Company, New York, (1960).

[13] J. P. Singhal and H. M. Srivastava, A class of bilateral generating functions for certain classical polynomials, Pacific J. Math., 42 (1972), pp. 755-762. http://dx.doi.org/10.2140/pjm.1972.42.755 
[14] H. M. Srivastava and H. L. Manocha, A Treatise on Generating Functions, Halsted Press, John Wiley \& Sons, New York, (1984).

[15] H. M. Srivastava and P. W. Karlsson, Multiple Gaussian hypergeometric series, Halsted Press, John Wiley \& Sons, New York, (1985).

Received: April 11, 2014; Published: November 19, 2014 